treated chicks-associated with an increased ability of the membrane to take up calcium-were an increase in the $\omega: 6$ fatty acids linoleic and arachidonic, and a decrease in at least one of the $\omega: 3$ series, docosahexaenoic acid.

The functions of the two dihydroxy metabolites $24,25(\mathrm{OH})_{2} \mathrm{D}_{3}$ and 25,26 $(\mathrm{OH})_{2} \mathrm{D}_{3}$ are still far from being resolved. J. A. Kanis (Nuffield Orthopaedic Centre, Oxford) suggested that $24,25(\mathrm{OH})_{2} \mathrm{D}_{3}$ may act directly on bone to increase mineralisation, on the basis of the observation that $1 \mu \mathrm{g} \mathrm{d}^{-3}$ of $24,25(\mathrm{OH}) \mathrm{D}_{3}$ ( $\mathrm{R}+\mathrm{S}$ epimers) administered to an anephric patient with osteomalacia, increased intestinal calcium absorption and reduced plasma alkaline phosphatase, without causing an increase in plasma or urine calcium. These effects were not observed by A. M. Pierides (University of Newcastle upon Tyne) however, in patients receiving as much as $16 \mu \mathrm{g} \mathrm{d}^{-3}$ of $24,25(\mathrm{OH})_{2} \mathrm{D}_{3}$.

The most ignored dihydroxy metabolite of vitamin $\mathrm{D}$ is certainly 25 , $26(\mathrm{OH})_{2} \mathrm{D}$. Very little is known about its properties and its in vivo origin is completely unknown. That it is a significant metabolite in man at least, was clearly demonstrated by E. B. Mawer (University of Manchester). In vitamin $D$ replete individuals the serum concentrations of $25,26(\mathrm{OH})_{2} \mathrm{D}$ and 24 , $25(\mathrm{OH}): \mathrm{D}$ are similar. Whereas the production of $1,25(\mathrm{OH})_{2} \mathrm{D}_{3}$ and 24,25$(\mathrm{OH})_{2} \mathrm{D}_{3}$ are governed by parameters such as the state of vitamin D nutrition and serum calcium concentration, no such controls are evident for 25,26$(\mathrm{OH})_{2} \mathrm{D}_{3}$ synthesis.

The papers referred to were only a few of the highlights of this workshop. But they do indicate that the next decade should be fruitful. By the 1980s the functions and mechanisms of action of all the vitamin D metabolites should be known, and the importance of 1 , $25(\mathrm{OH}) \mathrm{D}$ in phosphate regulation should be determined. The answers to these questions should provide reliable therapeutic procedures for the treatment of clinical disorders such as the renal osteodystrophies and postmenopausal osteoporosis.

\title{
Minicomputers for quantum chemists
}

\section{from Graham Richards}

QuANTuM chemists have always been major users of the most powerful computing facilities. Indeed, in recent years, the advances in the subject have followed the introduction of each new generation of machines and the capability of doing the best work has, to a large extent, been limited to those with access to major computing centres. As a result the subject has been dominated by a small number of groups and not used as a tool by more applied chemists to study or clarify their own research problems. The rapidly falling cost and increasing power of minicomputers should lead to a radical change if it were possible to perform the massive $a b$ initio quantum mechanical calculations on minicomputers rather than giant number-crunchers. A pilot study which succeeds in getting the quart into a pint pot and studying genuinely interesting problems has been conducted in the Chemistry Department in Berkeley.

The question "are minicomputers suitable for large scale scientific computation?" was answered in the affirmative by Schaefer in a paper presented at the IEEE Computer Society Conference held in Washington in September 1975 and in the proceedings of that meeting the relative capabilities and suitability of a number of minicomputers are compared. Since that date memory prices have fallen and new developments such as high speed array processors make the use of the minicomputer a very attractive alternative to the use of expensive large machines.

Calculations may take of the order of 30 to 40 times as long on a minicomputer as they do on a large scientific machine. However, since the calculations may be run overnight and the machine can be operated by graduate students or research personnel rather than professional operators, it may be much cheaper to work in this way.

The molecular structure calculations which have been attempted using a minicomputer have not been limited to the study of simple electronic ground states of closed-shell molecules. The Berkeley group has studied the excited electronic states of both glyoxal and ketene (J. Am. chem. Soc. 98, 401, $2689 ; 1976$ ). For each of the molecules, some 20 excited states are included and details are given of geometries as well as excitation energies.

The same minicomputer provided the means of studying some fundamental chemical reactions with $a b$ initio molecular orbital calculations. Two reactions involving methylene have formed the subject of articles in the Journal of the American Chemical Society (98, 1653, 3073: 1976). For the least-motion insertion reaction of methylene and hydrogen to yield methane the transition state geometry and activation energy were calculated and the abstrac- tion reactions of triplet methylene with hydrogen and methane studied in the same way. For the methane abstraction

$$
\mathrm{CH}_{4}\left({ }^{3} \mathrm{~B}_{1}\right)+\mathrm{CH}_{4} \rightarrow \mathrm{CH}_{3}+\mathrm{CH}_{3}
$$

the work implies that the reaction should not occur to a significant degree at room temperature contrary to the predictions of Dewar based on his semi-empirical MINDO calculations.

Although it is still early to be sure that the minicomputer will lead to a welcome change of life for quantum chemists it is striking just how wide is the range of problems tackled to date with the relatively cheap form of computer. Not only have quantum mechanical calculations been performed, but semiclassical computations. such as atomic scattering from solid surfaces, have also been studied in this way; an example being the scattcring of helium and neon from tungsten, the work of Miller and his colleagues $(J$. chem. Phys. 64, 45; 1976).

If quantum chemistry can be made inexpensive enough for chemists in experimental research laboratories to run their own calculations then the day may at last come when experimentalists can run their own calculations routincly. This will save the literature heing over-burdened with details of computations which may be directly interesting only to a small audience, and will enable practical chemists, such as medicinal chemists, to reap the benefit of useful calculations without needing to persuade a theoretical group to take on their work.

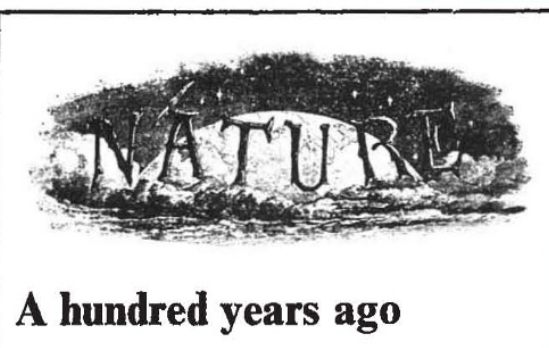

THE total expenditure on the new building at South Kensington for the reception of the Natural History Collections now in the British Museum is stated in the new Civil Service Estimates to have been 206,472I. up to September 30 last. A further sum of $36.650 \mathrm{l}$. is required to carry on the works up to the end of the present financial ycar. This amount has been already voted. The proposed vote for the present financial year $1877-78$ is $70,000 \mathrm{l}$, leaving the amount of $1,878 \mathrm{l}$. necessary to completc the building, the total estimate having been $395.000 \mathrm{l}$. We may remark that it is not only in this country that a new Museum of Natural History is in progress. Both at Paris and at Berlin the present buildings for the National Museum are found to be too small, and large sums arc to be appropriated to their reconstruction.

From Nature 15, March 1, 382; 1877 